\title{
Update on Basic Research into Superconducting Maglev and Research on Application of Maglev Technology to Conventional Railway Systems
}

\author{
Ken NAGASHIMA \\ Maglev Systems Technology Division
}

\begin{abstract}
RTRI is advancing basic research into superconducting maglev. The topics covered in this field include experimental evaluation of REBCO high-temperature superconducting coils, the development of a system for collecting sensor data from ground coils, inter alia. RTRI is also conducting research for the application of maglev technology to conventional railway systems. The areas covered in this field are wireless power transfer systems and flywheel energy storage systems.
\end{abstract}

Keywords: maglev, conventional railway, superconducting magnet, ground coil, wireless power transfer, flywheel energy storage system

\section{Introduction}

The development of superconducting magnetically levitated transportation system technology has been conducted as part of the Master-plan for Superconducting Maglev Technological Development, which was jointly prepared by JR Central and RTRI based on the relevant circular notice issued in 1990 by the then Minister of Transport.

RTRI's Maglev Systems Technology Division has been advancing the development of Maglev technology in line with this master plan. The Division has also been studying possible applications to conventional railway of superconducting, low-temperature, linear motor and other technologies that have been reaped from the research and development for maglev systems.

This paper discusses the current status of research and development on maglev systems and the application of related technologies to the conventional railway.

FY 2018 saw two encouraging developments for the Maglev Systems Technology Division. One of them concerns a high temperature superconducting magnetic system using rare earth (REBCO) high temperature superconducting wire that had been under development for more than a decade as part of the basic research on Maglev. A real-scale coil was successfully assembled onto the system. The other concerns an agreement that was reached with a railway operator to test a superconducting flywheel energy storage system on a commercial line. The system had been under development for more than a decade as part of research on possible applications of superconducting FESS to conventional railways.

\section{Basic research on maglev systems at RTRI}

\subsection{Development of a high temperature supercon- ducting magnet}

RTRI has been advancing research and development on the application to superconducting magnets of high temperature superconducting wire, especially rare earth versions that potentially can offer high critical current density in a magnetic field and at the same time be available at reduced cost.

Today, many companies engage in the production or development of rare earth high temperature superconducting wire, trying to offer their products at relatively low prices. Japanese companies doing this include Furukawa Electric (production by SuperPower of the US), Fujikura, SWCC Showa Cable Systems and Sumitomo Electric Industries. Overseas companies include Bruker HTS (Germany), SuNAM (Korea), AMSC (the US), SuperOX (Russia/ Japan) and Shanghai Superconductor Technology (China). The use of high temperature superconducting wire on a superconducting magnet eliminates the need for liquid helium and therefore expands the range of cooling options to choose from. Figure 1 shows an example of an ordinary superconducting magnet.

A two-stage cryocooler is used to cool the radiation heat shield in the first stage and the superconducting coil in the second stage. The cryocooler, which does not use refrigerants and therefore requires no filling and other related operations, can be started simply at the push of a button. However, the problem that it takes longer for the cryocooler to achieve a lower target temperature has to be overcome.

By adopting a design method that offers a higher operating temperature by taking advantage of the excellent critical current properties of rare earth high temperature superconducting wire in a high temperature, high magnetic field, the structure of the current superconducting magnet can be changed substantially with respect to the

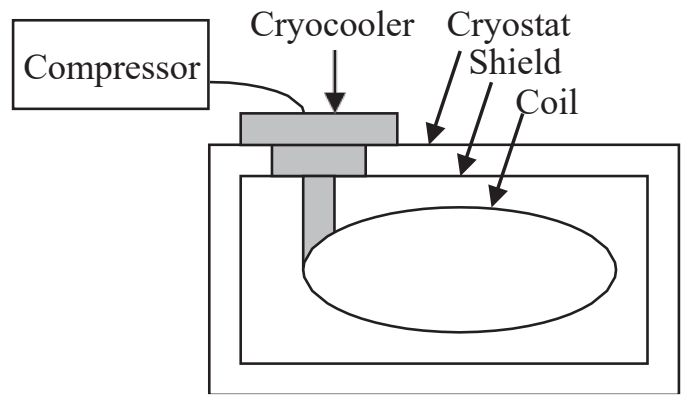

Fig. 1 High temperature superconducting magnet with a 2-stage cryocooler 


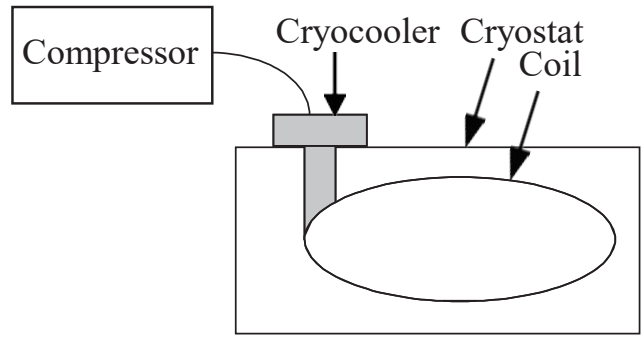

Fig. 2 High temperature superconducting magnet with a single stage cryocooler

following points:

- A highly efficient, single-stage cryocooler may be used.

- The shield for the superconducting coil can be eliminated.

- Then, the heat insulation can be eliminated.

Based on the above, the cross-sectional area of a superconducting magnet cryostat can be made smaller, which leads to a reduction in mass and production cost. The resultant superconducting magnet may look like the one in Fig. 2. With the elimination of the superconducting coil shield, the superconducting coil can be positioned closer to the ground coil, making it possible to increase the magnetic flux around the ground coil with the same magnetomotive force. If the magnetic flux does not need to be increased, then the magnetomotive force of the superconducting coil can be lowered, which allows the superconducting wire to be reduced in volume, allowing cost to be reduced.

RTRI plans to conduct electromagnetic vibration tests using a high temperature superconducting magnet of the above configuration to evaluate long-term durability of ground coils. The paper titled "Monitoring and Protection Method for a REBCO Magnet Designed for Electromagnetic Vibration Tests" in the present issue of QR, discusses a high temperature superconducting magnet (shown in Fig. 3), which was fabricated based on the concept in Fig. 2. Meanwhile, electromagnetic vibration tests currently conducted at RTRI use a low temperature superconducting magnet shown in Fig. 4. While these magnets were shot from different angles and therefore cannot be compared easily, the magnet in Fig. 3 does not have a refrigerant tank like the one in Fig. 4 or any internal piping to send a refrigerant from the tank to the coils. As a result, the magnet in Fig. 3 has a lower-profile outer tank (the thin rectangular parallelepiped at the bottom), which contains superconducting coils, is thinner as there is no shield inside and has a crosssection area about $40 \%$ smaller than the magnet in Fig. 4.

High temperature superconducting magnet conduction cooled by a cryocooler has proven to be easier to handle and have a shorter precooling time from room temperature than conventional low temperature superconducting magnets that use refrigerants such as liquid helium. The high temperature version will be tested further for reliability and durability through ground coil electromagnetic vibration tests and other methods.

Being a key component, a key goal is to develop a method for protecting the superconducting magnet in emergencies. To protect the superconducting magnet, it is important to monitor the conditions inside the supercon-

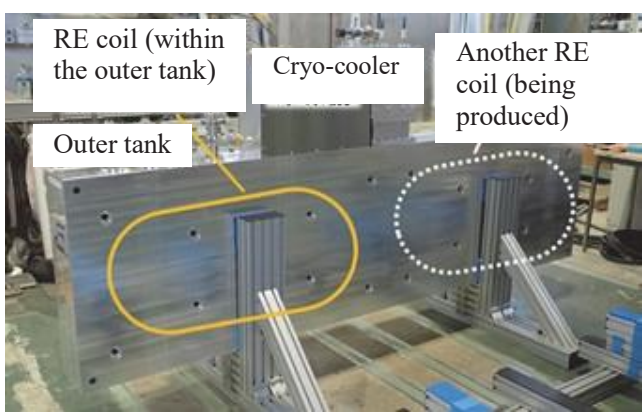

Fig. 3 High temperature superconducting magnet for electromagnetic vibration tests of ground coils

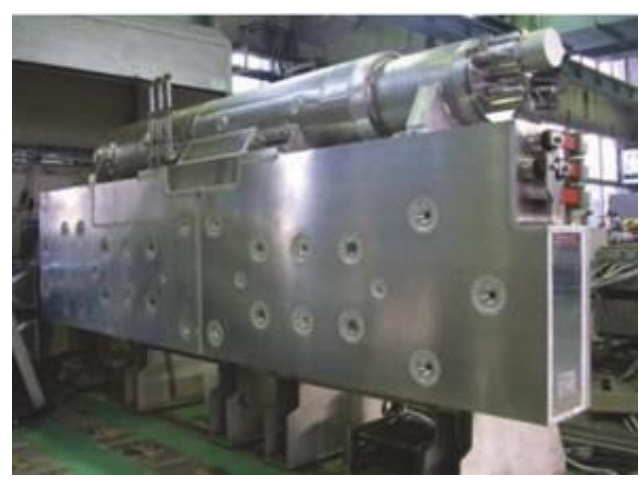

Fig. 4 Low temperature superconducting magnet for electromagnetic vibration tests of ground coils

ducting coil. This paper discusses both monitoring and the prospect of developing an emergency protection method.

The research and development activities described above were partly subsidized by the Ministry of Land, Infrastructure, Transport and Tourism to support the development of railway technologies.

\subsection{Research on ground coils}

Maglev involves a great number of ground coils installed outdoors and these coils are expected to last many years. There are two types of ground coil: the propulsion coil and the levitation and guidance coil. As a special high voltage component, the propulsion coil must have a stable insulation performance. However, insulation diagnosis in the field of propulsion coils, which form linear motors with superconducting magnets (coils), is difficult to perform unless the propulsion coils function as a load (counter electromotive force) as a train runs in order to receive the correct voltage from the substation.

The paper titled "Insulation Diagnosis with a Focus on Partial Discharge of the Propulsion Coils of the Superconducting Maglev" in the present issue of QR discusses a new method that enables field insulation diagnosis of propulsion coils in situ. The insulation diagnosis method is shown in Fig. 5. With high voltage applied to the propulsion coils, the vehicle is run to measure electromagnetic waves using dipole antennas and other inspection probes. Any detection of specific electromagnetic waves that suggest partial discharge in a section where propulsion coils are installed indicates the possibility that some of the propulsion coils in 


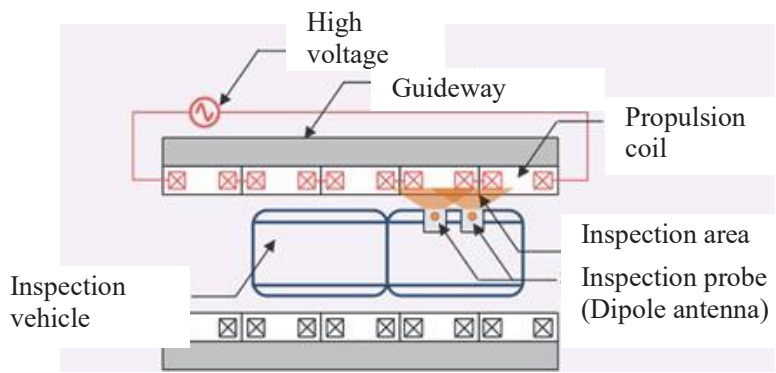

Fig. 5 Schematic of insulation diagnosis based on partial discharge

the section might have a reduced insulation performance. Using a number of antennas enables the calculation of differences in time taken for electromagnetic waves to reach different antennas, and the calculations can then be used to locate the propulsion coils that are partially discharged.

As indicated in this paper, future plans include quantitative evaluation of reduced insulation performance levels of propulsion coils and an experiment using the current collection test equipment at RTRI to detect electromagnetic waves emitted from discharge sources on the ground from a vehicle running at high speed.

Meanwhile, the RFID (Radio Frequency Identification) technology discussed in the paper titled "Report of 9th Annual IEEE International Conference on RFID Technology and Applications 2018 (RFID-TA 2018)" in the present issue of RTRI Report also relates to maintenance technology for ground coils. RTRI has been studying on its own the possibility of applying RFID technology to the maintenance including of ground coils for maglev systems. As part of the study, RTRI proposed an opportunistic communication technology which enables reading of tags on the move and tested its performance on the Miyazaki maglev test track using a vehicle with an onboard reader/writer and antenna simulating a maintenance car. The test found that data can be gathered on the move, that maintenance data can be written on tags and that condition monitoring can be performed using a tag sensor, which combines the functions of a sensor and a tag.

This technology can be exploited extensively for infrastructure maintenance of not just maglev systems but also conventional railways, and therefore will be researched further for application in those areas.

\section{Research on the application of related technolo- gies to conventional railways}

\subsection{Wireless power transfer technology}

RTRI has been endeavoring to improve wireless power transfer technology, currently used for power transmission to the onboard receiving system for maglev systems, by reducing loss and increasing output and power transfer rates per unit area, so that the improved technology can also be used for hybrid railcars and other vehicles with an energy storage function to receive power including when they stop at a station (Fig. 6). The actions taken thus far include those aimed at reducing loss by adopting figure 8 coils and improving litz wire and experiments conducted on a test track at RTRI in 2014 to transfer power to the onboard receiving system. The paper titled "Design Study Aiming at Increasing Transfer Power per Unit Area of the Coil for the Wireless Power Transfer System of Railway Vehicles" in the volume 33, Number 5 of RTRI Report presents the results of an attempt to increase power transmission rates per unit area.

Related activities are underway overseas as well. In Augsburg, Germany, since 2010 running tests on the Primove system of Bombardier, a contactless power supply system, have been in progress using trams. In 2014, the Korea Railroad Research Institute conducted a demonstration test of a wireless power transfer system on its test track using HEMU (next-generation high speed railway) vehicles. In the automotive sector, a number of projects are underway to verify the performance of on-the-move electric vehicle charging systems. It has been reported that in 2013 experiments were conducted at the Korea Advanced Institute of Science and Technology (KAIST) and that in 2015 a test course embedded with a wireless power transfer system for automobiles was constructed at Utah State University.

While keeping up with these moves abroad, RTRI will continue with the development of wireless power transfer technology including the possibility of introducing it first to smaller-sized means of transport such as trams and BRT.

\subsection{Superconducting flywheel energy storage sys- tem}

Since its launch in 2005, the Maglev Systems Technol-

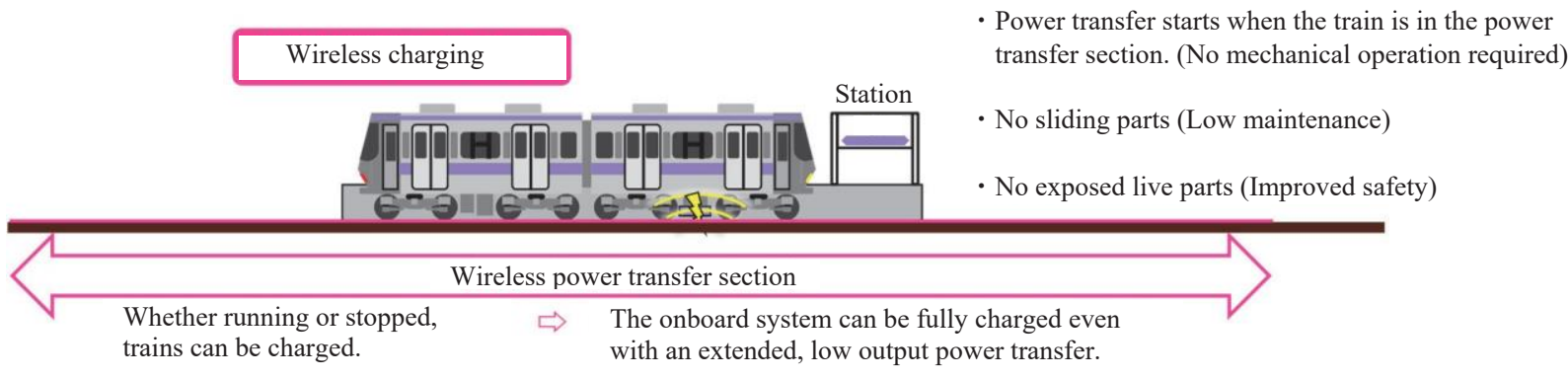

Fig. 6 Wireless power transfer 
ogy Division has been developing a superconducting flywheel energy storage system as a major application to the conventional railway. Along with this, since 2012 in a joint effort with Yamanashi Prefecture, manufacturers and other partners and with subsidies from NEDO, the Division has been developing a superconducting flywheel energy storage system. Building on the achievements from these efforts and to put a superconducting flywheel energy storage system to practical use for railway businesses, RTRI, Yamanashi Prefecture and the East Japan Railway Company (JR East) on March 29, 2018, signed a letter of intent concerning the development of superconducting flywheel energy storage system technology for railway applications. The tasks assigned to the three parties are shown in Fig. 7.

Railway operators generally install energy storage systems in substations primarily to deal with loss of regenerative energy and voltage drops as part of their business continuity planning (BCP).

While secondary batteries and capacitors have been used for these purposes in recent years, in 1981 Keikyu Corporation installed a flywheel energy storage system at the Seto substation in Kanazawa and tested the system, and in 1988 installed a FESS (output: 3 MW, capacity: 25 $\mathrm{kWh}$ ) in Zushi which was placed into commercial operation. This system was designed to deal with voltage dropping in the overhead contact line near the end of the Zushi branch line. Installed more than 30 years ago, the system is still being used. The flywheel is made of alloyed steel and weighs 13.7 tons. The flywheel shaft is horizontally installed and supported by two oil film bearings at both ends.

This flywheel appears to be the only flywheel for railway applications still working in Japan. Besides this, JR Central, commissioned by NEDO, developed a flywheel energy storage system (output: $1 \mathrm{MW}$, capacity: $50 \mathrm{kWh}$ ) for railway applications. The metal flywheel was supported by the attractive force of low temperature superconducting (NbTi) coils and iron. Outside Japan, the Administrator of Railway Infrastructures (Adif) in Spain developed an energy storage system (output: $350 \mathrm{~kW}$, capacity: $55.5 \mathrm{kWh}$ ) that houses a metal flywheel supported by magnetic bearings using normal magnets. Similarly, Stornetic of Germany posts on their web site an energy saving system (output: $22 \mathrm{~kW}$, capacity: $3.6 \mathrm{kWh}$ ) that houses a metal flywheel supported by magnetic bearings using normal magnets. The company says the system can also be used for railway applications. Neither of these systems however, appear to have been ready for practical use. With the Stornetic system, as the capacity is smaller than the other systems, a number of units likely may be combined for operation. Reviewing the specifications of these systems gives an idea of system requirements for railway applications. The output would be from several hundred $\mathrm{kW}$ to several MW. The capacity would be from around $25 \mathrm{kWh}$ to around $50 \mathrm{kWh}$. The Stornetic system can meet these requirements when ten units are combined.

In further pursuing the current RTRI project, it has been decided to focus on the existing technologies that have proven successful for possible adoption given that safety and reliability come first in the railway business, unless contactless superconducting magnetic bearings need to be employed to eliminate frictional wear of bearings and resultant loss and reduce maintenance.

For these reasons, alloyed steel has been chosen as the material for the flywheel, a key component of the system. The energy that can be stored by the flywheel can be increased in two ways: increasing its weight and increasing its speed. The stored energy is proportional to the weight and proportional to the square of the speed. To increase the speed, however, the flywheel needs to be stronger which makes it more expensive. In addition, high-speed generator motors that also have a high output, a requirement for railway applications, are currently not commercially available. Consequently, a flywheel similar in size to that of the performance-proven Keikyu Corporation system, appears to be more practical.

The superconducting magnetic bearing proposed by RTRI is capable of supporting heavy loads. Due in part to the improved performance of superconducting wire, the

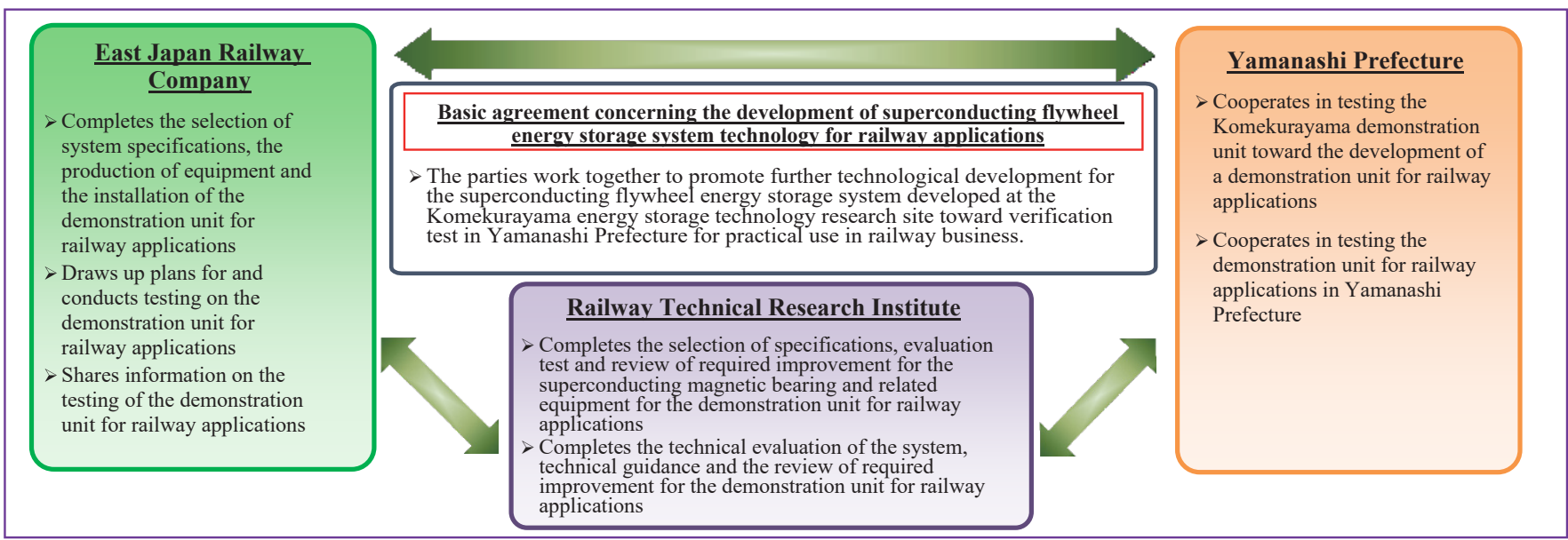

Fig. 7 Tasks assigned to the parties toward putting the superconducting flywheel energy storage system to practical use in railway business 
anticipated load for railway applications has been found to require only a slight increase in superconducting wire and bulk. This is detailed in the paper titled "Verification of the Reliability of the Superconducting Magnetic Bearing in a Flywheel Energy Storage System for its Application to Railways" in the present issue of QR. Besides this paper which presents conservative evaluations, the paper titled "Key Technology of the Superconducting Flywheel Energy Storage Demonstration Machine" in volume 33, Number 5 of the RTRI Report presents the development and reliability verifications of peripheral equipment that would be required, as well as related principles of operation, functions and other subjects.

\section{Author}

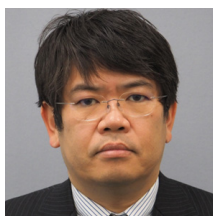

Ken NAGASHIMA, Dr. Eng

Director, Head of Maglev Systems Technology

Division

Research Areas: Superconducting

Technologies, Cryogenics

\section{Conclusion}

As researchers, our ultimate goal of basic research is that homegrown maglev system technologies will be used on the Yamanashi maglev test line and/or on the upcoming commercial line (Linear Chuo Shinkansen). Equally, our goal of research on the application of maglev technology to conventional railway systems is the use of these technologies going into verification tests and successfully put into practical use with railway operators. As the results of this research begin to take shape, our resolve to work even harder to build on these successes is even stronger. 